\title{
Avaliação de métodos de detecção da fosfatase alcalina em leite bovino, bubalino e caprino
}

\author{
Evaluation of alkaline phosphatase detection methods in \\ bovine, buffalo and goat milk
}

Joelson Sousa Lima1* (1), Ana Paula Presley Oliveira Sampaio², Poliana Santos Silva ${ }^{3}$, Mylla Christy da Silva Dufossé ${ }^{1}$, Adrianne Maria Brito Pinheiro da Rosa², Carina Martins de Moraes ${ }^{2}$, Talita Bandeira Roos ${ }^{1}$

${ }^{1}$ Universidade Federal do Pará (UFPA), Instituto de Medicina Veterinária, Faculdade de Medicina Veterinária de Castanhal, Laboratório de Microbiologia e Imunologia, Castanhal/PA - Brasil

${ }^{2}$ Universidade Federal do Pará (UFPA), Instituto de Medicina Veterinária, Faculdade de Medicina Veterinária,

Laboratório de Higiene e Qualidade de Alimentos, Castanhal/PA - Brasil

${ }^{3}$ Universidade do Estado do Pará (UEPA), Marabá/PA - Brasil

*Corresponding Author: Universidade Federal do Pará (UFPA), Instituto de Medicina Veterinária, Faculdade de Medicina Veterinária de Castanhal, Laboratório de Microbiologia e Imunologia, Campus de Castanhal-II, BR 316 km 62, Bairro Saudade, CEP: 68746-000, Castanhal/PA, Brasil, e-mail: joelsonbio@live.com.

Cite as: Lima, J. S. Sampaio, A. P. P. O., Silva, P. S., Dufossé, M. C. S., Rosa, A. M. B. P., Moraes, C. M., \& Roos, T. B. (2021). Evaluation of alkaline phosphatase detection methods in bovine, buffalo and goat milk. Brazilian Journal of Food Technology, 24, e2020130. https://doi.org/10.1590/1981-6723.13020

\begin{abstract}
Resumo
A pasteurização compreende uma etapa de suma importância para eliminação de patógenos no leite e, a fim de manter a inocuidade do alimento, o controle desse processo é fundamental do ponto de vista da segurança alimentar. Assim, o objetivo deste trabalho foi verificar a aplicabilidade e a sensibilidade dos procedimentos de detecção da fosfatase alcalina em leite bovino, bubalino e caprino, experimentalmente pasteurizados e fraudados com alíquotas de leite cru. O leite foi pasteurizado e acrescido de leite cru nas seguintes percentagens: $0,1 \% ; 0,25 \%$; $0,5 \% ; 0,75 \% ; 1 \% ; 3 \% ; 5 \%$ e $7 \% ; 10 \% ; 50 \%$, além da adoção dos controles: negativo (leite pasteurizado) e positivo (leite cru). Os métodos utilizados foram: a metodologia oficial da legislação brasileira, tiras colorimétricas e kit de reagentes. Os resultados demonstraram que nos testes realizados com leite bubalino houve uma discreta reação quando utilizados o kit de reagentes e as tiras colorimétricas, ao passo que a metodologia segundo a legislação se apresentou pouco eficiente. Para o leite caprino a enzima não foi detectada nas amostras de leite testadas por nenhuma das metodologias adotadas. Já para o leite bovino o procedimento mais sensível para detectar porções mínimas de leite cru foi o da legislação brasileira. A metodologia da legislação brasileira é a que possui maior sensibilidade para acusar a presença da fosfatase alcalina no leite bovino, e os procedimentos para o controle da pasteurização em leite bovino não possuem a mesma eficácia para os leites bubalino e caprino que, neste caso, precisam ser otimizadas.
\end{abstract}

Palavras-chave: Pasteurização; Controle de qualidade; Segurança alimentar; Fraude; Métodos físico-químicos; Lácteos. 


\begin{abstract}
Pasteurization is a very important step for eliminating pathogens in milk and keeping the food-safe, the control of this process is fundamental from the point of view of food safety. Thus, the objective of this work was to verify the applicability and sensitivity of the alkaline phosphatase detection procedures in bovine, buffalo, and goat milk, experimentally pasteurized and defrauded with raw milk aliquots. The milk was pasteurized and added raw milk in the following percentages: $0.1 \% ; 0.25 \% ; 0.5 \% ; 0.75 \% ; 1 \% ; 3 \% ; 5 \%$ and $7 \%, 10 \%, 50 \%$, besides the adoption of the controls: negative (pasteurized milk) and positive (raw milk). The methods used were: the official methodology of the Brazilian legislation, colorimetric strips, and a reagent kit. The results showed that in the tests performed with buffalo milk, there was a slight reaction when using the reagent kit and the colorimetric strips, while the methodology according to the legislation was inefficient. For goat milk, the enzyme was not detected in the milk samples tested by any of the adopted methodologies. For bovine milk, the most sensitive procedure to detect minimal portions of raw milk was the Brazilian legislation. The methodology of the Brazilian legislation is the one with the highest sensitivity to acknowledge the presence of alkaline phosphatase in bovine milk and the procedures for the control of pasteurization in bovine milk do not have the same efficacy for buffalo and goat milk that, in both it is necessary to be optimized.
\end{abstract}

Keywords: Pasteurization; Quality control; Food safety; Fraud; Physical-chemical methods; Dairy.

\title{
1 Introdução
}

O leite é um alimento que apresenta elevado valor nutricional, sendo apreciado por grande parte da população. No entanto, devido a sua composição, torna-se um produto altamente perecível. Por essa razão é necessário que sua produção e manipulação sejam executadas com um controle de qualidade eficiente, em especial no processamento da matéria-prima na indústria de alimentos. Nesse sentido, as análises físicoquímicas e microbiológicas são indispensáveis para que se garanta a qualidade do leite e de seus derivados (Rosa-Campos et al., 2011; Fracasso \& Pfüller, 2014).

Atualmente, além do bovino, o leite de outras espécies, como o bubalino e o caprino, tem despertado o interesse dos consumidores. O leite bubalino possui elevado valor nutricional, devido ao considerável teor de proteínas, minerais, gordura e baixos níveis de colesterol, características estas que contribuem para uma dieta saudável (Araújo et al., 2011; Pignata et al., 2014). Já o leite de cabra, além de possuir altas taxas de nutrientes, representa uma alternativa para pessoas com alergia ao leite bovino, o que chama a atenção de diversos produtores em várias regiões do país (Jirillo et al., 2010).

Nesse sentido, devido à abertura do mercado e diante de uma nova proposta de consumo, que abrange o leite de outras espécies, é necessária a garantia da seguridade do alimento produzido. No entanto, os critérios adotados para a realização dos testes de qualidade do produto baseiam-se exclusivamente no leite de origem bovina, sem levar em conta as características bioquímicas de outros tipos de leites, como o de búfala e o de cabra. Assim, é de extrema relevância avaliar se as metodologias adotadas se aplicam aos leites bubalino e caprino, que têm tido uma ampla aceitação e consumo, a fim de garantir a inocuidade desse alimento.

A pasteurização é um procedimento fundamental para a eliminação dos agentes patogênicos do alimento, tornando-o um produto inócuo, alterando o mínimo possível a sua qualidade nutricional, química e sensorial (Antunes, 2014), sendo dessa forma essencial para garantir a seguridade microbiológica dos produtos lácteos disponíveis comercialmente. Uma das formas de controle da pasteurização baseia-se na detecção da enzima fosfatase, que pode indicar tanto falhas no processo térmico quanto fraude por adição do leite cru ao pasteurizado, tratando-se de um teste físico-químico grande interesse na prática rotineira de laticínios (Brasil, 2006, 2011). A enzima é naturalmente encontrada no leite in natura, porém inativada com o processamento térmico eficiente, dentro dos padrões de tempo e temperatura adequados (Almeida, 2006; Silva et al., 2009; Egger et al., 2016). 
Após o processamento térmico são utilizados testes que verificam a atividade residual da fosfatase alcalina, a fim de constatar se binômio tempo-temperatura foi eficiente para a pasteurização do produto. Além do procedimento oficial determinado na legislação brasileira (Brasil, 2006), kits de reagentes e fitas colorimétricas podem ser utilizados para a verificação da qualidade do processamento térmico por meio da detecção da fosfatase alcalina. Porém, poucos trabalhos investigam a sensibilidade desses métodos (Seixas et al., 2014) e se são aplicáveis em outros tipos de leite, uma vez que foram elaborados tendo como parâmetro o leite bovino.

Assim, o objetivo deste trabalho foi verificar a aplicabilidade e a sensibilidade dos procedimentos de detecção da fosfatase alcalina em leites bovino, bubalino e caprino experimentalmente pasteurizados e fraudados com alíquotas de leite cru, em diferentes porcentagens.

\section{Material e métodos}

O presente estudo foi realizado no laboratório de Higiene e Qualidade de Alimentos, do Instituto de Medicina Veterinária da Universidade Federal do Pará - Campus de Castanhal. As amostras de leites bovino, bubalino e caprino foram experimentalmente pasteurizadas, separadas em aliquotas e fraudadas com leite cru.Os testes foram realizados sempre em triplicata, para garantir maior exatidão na avaliação dos resultados.

Para o processamento térmico, $600 \mathrm{~mL}$ do leite de cada espécie foram pasteurizados em banho-maria, durante 15 segundos, a $72{ }^{\circ} \mathrm{C}$, seguido de resfriamento imediato a $37{ }^{\circ} \mathrm{C}$. Em seguida, procedeu-se a incorporação do leite cru nas seguintes percentagens: $0,1 \% ; 0,25 \% ; 0,5 \% ; 0,75 \% ; 1 \% ; 3 \% ; 5 \%$ e $7 \% ; 10 \%$; $50 \%$. Além disso, também foram realizados os controles negativo (com o leite pasteurizado e não fraudado) e positivo (leite cru).

As amostras de leite previamente pasteurizadas e adulteradas foram submetidas a 3 diferentes metodologias de verificação da atividade enzimática da fosfatase alcalina, denominadas em nosso trabalho de metodologias A, B e C. A metodologia " $A$ " foi a preconizada pela Instrução Normativa n ${ }^{\circ}$ 62, de 29 de dezembro de 2011, do Ministério da Agricultura, Pecuária e Abastecimento (Brasil, 2011). Já a metodologia "B" baseou-se no uso de tiras colorimétricas e a "C" na utilização de um kit comercial. O fundamento da técnica para a deteç̧ão da enzima, nos três tratamentos, baseia-se na adição de um substrato específico que, em contato com a enzima, é degradado e os produtos são revelados através de um indicador colorimétrico, e os protocolos adotados para cada metodologia seguiram as recomendações da legislação brasileira (metodologia A) e dos respectivos fabricantes (metodologias B e C).

\section{Resultados e discussão}

A detecção da fosfatase alcalina no leite bovino foi realizada com grande desempenho quando se empregou a metodologia " $\mathrm{A}$ " em todos os tratamentos realizados, a partir do menor percentual de fraude com leite cru. Contudo, as demais metodologias (B e C) só demonstraram eficiência em acusar a presença da enzima nas amostras com o incremento superior a $2 \%$ do leite não pasteurizado.

Essa informação corrobora os resultados obtidos por Seixas et al. (2014), que avaliaram o desempenho de tiras colorimétricas e de um kit de reagentes para detecção da enzima em diferentes proporções de leite cru bovino (1\% a 10\%) incorporado ao leite pasteurizado. Os resultados desses autores mostraram que os testes comerciais não foram capazes de detectar a presença da fosfatase alcalina em leite pasteurizado, contaminado com quantidades inferiores a $2 \%$ de leite cru, o que pode apresentar um resultado falso negativo se o percentual de fraude estiver sido abaixo desse valor.

Neste caso, embora a metodologia proposta pela legislação necessite de equipamentos e tempo maior para a execução quando comparada com os outros dois métodos, que são mais práticos e com leitura imediata, ainda assim é o de maior eficiência e sensibilidade para a detecção de percentagens mínimas da enzima no leite bovino. 
Franco et al. (2011) avaliaram a qualidade do leite bovino cru, pasteurizado e longa vida, analisando a acidez e a atividade das enzimas peroxidasse e fosfatase. Para detecção da fosfatase, os autores utilizaram o método descrito pela legislação e os resultados obtidos demostraram a ausência da enzima nas amostras processadas termicamente. Tais dados ratificam a eficiência da metodologia proposta pela legislação brasileira para análise do leite bovino.

Outros estudos com o objetivo de detectar a enzima fosfatase alcalina a partir de diferentes técnicas já foram realizados em leite cru bovino (Machado et al., 2009; Mata et al., 2012), em função de este ser um produto amplamente consumido. Porém, poucas pesquisas tiveram como objetivo comparar métodos de detecção da fosfatase alcalina e, principalmente, a sensibilidade desses procedimentos na identificação de pequenas quantidades de leite cru incorporadas ao leite pasteurizado. Da mesma forma, estudos com leite de outras espécies como a de bubalinos e caprinos também são escassos e, portanto, se fazem necessários para que as metodologias que vem sendo aplicadas em nível de fiscalização possam ser utilizadas como referência para garantia do controle desse alimento (Sosnowski et al., 2016).

Para o leite bubalino os resultados deste trabalho demonstraram que as fitas colorimétricas só foram capazes de acusar a presença da enzima nas amostras contendo a partir de 3\% de leite bubalino cru. Além disso, com o kit, a fosfatase também foi detectada nas amostras contendo a partir de $3 \%$ de incorporação, sendo perceptível uma coloração mais intensa nos tratamentos com mais de 5\% de leite não pasteurizado. Quando os procedimentos da legislação foram adotados, os resultados não foram eficazes nem mesmo com os controles. Repetindo-se esse mesmo ensaio com outra amostra de leite, porém, foi perceptível uma leve alteração da tonalidade em algumas amostras, o que pode estar ligado à quantidade da enzima disponível no leite ou devido à interferência de outros constituintes na reação. Contudo, mais estudos são necessários para otimizar a detecção da fosfatase no leite bubalino, visto que ainda há uma escassez de dados na literatura sobre o controle da pasteurização com a pesquisa da enzima nesse tipo de leite.

No leite caprino, a fosfatase alcalina não foi detectada nas amostras analisadas por nenhuma das metodologias adotadas. Não houve reação característica para a positividade da enzima nas amostras fraudadas com leite cru caprino e nem nos tratamentos de controle deste. Logo, os procedimentos utilizados não são eficientes para a pesquisa da enzima em leite de cabra e, por conseguinte, para a avaliação da eficiência do processamento térmico nesse produto.

Giombelli et al. (2011), em seu estudo sobre avaliação de qualidade do leite bovino, a partir de testes físico-químicos e microbiológicos, em amostras de leite produzidas no estado do Paraná, relataram a presença da enzima fosfatase alcalina, o que provavelmente indica uma falha no processo térmico que pode tornar o leite uma possível fonte de contaminação, principalmente por agentes patogênicos. Com relação ao presente estudo, a impossibilidade de as técnicas adotadas detectarem a enzima no leite caprino e a incapacidade das metodologias acusarem a incorporação de menos de 3\% de leite cru bubalino podem implicar na avaliação da qualidade higiênico-sanitária do leite, que pode ter sido pasteurizado de forma inadequada e acabar acarretando danos à saúde do consumidor.

Outros trabalhos também já evidenciaram a ineficiência dos procedimentos de detecção da fosfatase alcalina quando aplicados em leite de cabra (Lima, 1988; Andrade et al., 2008). Essas pesquisas, bem como os resultados do presente trabalho, mostram que o uso das metodologias para o leite bovino não é eficaz em leite caprino. Assim, a não detecção da enzima não significa a ausência da mesma nas amostras de leite. Acredita-se que esses resultados imprecisos ocorrem pela baixa concentração da enzima no leite caprino, quando comparado ao bovino. Desse modo, não é possível distinguir os leites crus dos pasteurizados e nem aqueles em que houve falha no processamento térmico (Lima, 1988).

Essa informação corrobora com os dados descritos por Sosnowski et al. (2016) que afirmam que a quantidade de fosfatase alcalina é inferior no leite caprino em comparação ao leite bovino. Esses autores determinaram a atividade residual da enzima através de um método fluorimétrico em leite e queijo de cabra 
e ressaltaram a necessidade do uso de outros procedimentos para a análise da fosfatase alcalina no leite de cabra. Além disso, os autores relatam a escassez de trabalhos relacionados a esse tema.

O controle do processamento térmico é de fundamental importância na indústria de alimentos para a garantia da qualidade do produto final. Falhas nesses mecanismos ou mesmo a incorporação intencional de leite cru ao pasteurizado geram riscos à saúde pública, visto que pequenas proporções de leite cru podem ser responsáveis por veicular micro-organismos que são potenciais agentes causadores de enfermidades. Conforme descrito por Seixas et al. (2014), a inclusão de apenas 1\% de leite cru pode promover a proliferação de cerca de $12 \times 10^{5} \mathrm{UFC} / \mathrm{mL}$, valor acima ao estabelecido pela legislação que é $600 \mathrm{mil} \mathrm{UFC} / \mathrm{mL}$. A exemplo disso, Silveira \& Bertagnolli (2014) realizaram um trabalho com amostras de leite cru comercializado ilegalmente em feiras livres e demonstraram a presença de micro-organismos mesófilos, coliformes totais e termotolerantes acima do limite máximo estabelecido pela legislação brasileira, enfatizando o perigo relacionado ao leite cru.

\section{Conclusão}

Dessa forma, concluiu-se que as metodologias para a detecção da fosfatase alcalina no leite bubalino e caprino não apresentam a mesma eficiência que demonstraram ao serem aplicadas no leite bovino e precisam ser otimizadas para garantir a qualidade do produto e a segurança alimentar dos consumidores. Assim, é necessário o estabelecimento de outros mecanismos ou a otimização dos que já existem para a investigação da enzima visando a garantia da qualidade do produto processado. Porém, no leite bovino os procedimentos da legislação brasileira possuem maior sensibilidade por acusar baixas concentrações da enzima no alimento.

\section{Referências}

Almeida, A. O. (2006). Controle rápido da eficiência e segurança do processo de pasteurização do leite (HTST - High Temperature Short Time) (Dissertação de mestrado). Universidade Estadual Paulista, Jaboticabal.

Andrade, P. V. D., Souza, M. R., Penna, C. F. A. M., \& Ferreira, J. M. (2008). Características microbiológicas e físico-químicas do leite de cabra submetido à pasteurização lenta pós-envase e ao congelamento. Ciência Rural, 38(5), 1424-1430. http://dx.doi.org/10.1590/S0103-84782008000500036

Antunes, V. C. (2014). Uso de microfiltração para melhoria da qualidade e extensão da vida de prateleira de leite pasteurizado. Brazilian Journal of Food Technology, 17(1), 75-86. http://dx.doi.org/10.1590/bjft.2014.011

Araújo, T. P. M., Rangel, A. H. N., Soares, A. D., Lima, T. C. C., Lima Júnior, D. M., \& Novaes, L. P. (2011). Influência das estações do ano sobre a composição do leite de búfalas mantido em tanque de resfriamento. Agropecuária Científica no Semiárido, 7(1), 1-5.

Brasil. Ministério da Agricultura, Pecuária e Abastecimento. (2006, dezembro 12). Oficializa os métodos analíticos oficiais físicoquímicos, para controle de leite e produtos lácteos (Instrução Normativa №68). Diário Oficial [da] República Federativa do Brasil, Brasília.

Brasil. Ministério da Agricultura, Pecuária e Abastecimento. (2011, dezembro 11). Aprova o Regulamento Técnico de Produção, Identidade e Qualidade do Leite tipo A, o Regulamento Técnico de Identidade e Qualidade de Leite Cru Refrigerado, o Regulamento Técnico de Identidade e Qualidade de Leite Pasteurizado e o Regulamento Técnico da Coleta de Leite Cru Refrigerado e seu Transporte a Granel, em conformidade com os Anexos desta Instrução Normativa (Instrução Normativa $n^{\circ}$ 62). Diário Oficial [da] República Federativa do Brasil, Brasília.

Egger, L., Nicolas, M., \& Pellegrino, L. (2016). Alkaline phosphatase activity in cheese as a tracer for cheese milk pasteurization. Lebensmittel-Wissenschaft + Technologie, 65, 963-968. http://dx.doi.org/10.1016/j.lwt.2015.09.033

Fracasso, R., \& Pfüller, E. E. (2014). Processamento do leite para a fabricação do queijo na indústria de laticínios Camozzato Ltda, Sananduva - RS. RAMVI, Getúlio Vargas, 1(2), 713-720.

Franco, B. S., Manfio, S. R., De Andrade, C. J., \& Leão, M. F. (2011). Análise das enzimas peroxidase e fosfatase em amostras de leite cru, pasteurizado e longa vida. Revista Citino, 1(1), 52-56.

Giombelli, C. J., Tamanini, R., Bataglini, A. P. P., Magnani, D. F., Angela, H. L., \& Beloti, V. (2011). Avaliação da qualidade microbiológica, físico-química e dos parâmetros enzimáticos de leite pasteurizado e leite tipo $\mathrm{B}$, produzidos no Paraná. Semina: Ciências Agrárias, 32(4), 1539-1546. http://dx.doi.org/10.5433/1679-0359.2011v32n4p1539

Jirillo, F., Jirillo, E., \& Magrone, T. (2010). Donkey's and goat's milk consumption and benefits to human health with special reference to the inflammatory status. Current Pharmaceutical Design, 16(7), 859-863. PMid:20388098.

http://dx.doi.org/10.2174/138161210790883688 
Lima, C. I. P. (1988). Caracterização dos diferentes tratamentos térmicos na inativação da fosfatase alcalina para a avaliação da eficiência da pasteurização do leite caprino (Dissertação de mestrado). Universidade Federal de Minas Gerais, Belo Horizonte.

Machado, G. M., Santos, A. L., Costa Júnior, L. C. G., Costa, R. G. B., \& Paiva, P. H. C. (2009). Fosfatase alcalina em leite e derivados: Aspectos teóricos e práticos. Revista do Instituto de Latícinios Cândido Tostes, 370(64), 22-28.

Mata, N. F., Toleto, P. S., \& Pavia, P. C. (2012). A importância da pasteurização: Comparação microbiológica entre leite cru e pasteurizado, do tipo B. Revista do Instituto de Latícinios Cândido Tostes, 67(384), 66-70. http://dx.doi.org/10.5935/22386416.20120010

Pignata, M. C., Fernandes, S. A. A., Ferrão, S. P. B., Faleiro, A. S., \& Conceição, D. G. (2014). Estudo comparativo da composição química, ácidos graxos e colesterol de leites de búfala e vaca. Revista Caatinga, 27(4), 226-233.

Rosa-Campos, A. A., Borgo, L. A., Oliveira, Y. M. S., Rocha, J. E. S., Souza, L. M. J., \& Mendonça, M. A. (2011). Qualidade físico-química e microbiológica de leite tipo $\mathrm{C}$ produzido no distrito federal. Revista do Instituto de Latícinios Cândido Tostes, 66(378), 11-16.

Seixas, F. N., Fagnani, R., Rios, E. A., Pereira, J. R., Tamanine, R., \& Beloti, V. (2014). Comparação de métodos para detecção de fosfatase alcalina e peroxidase em leite. Revista do Instituto de Latícinios Cândido Tostes, 69(1), 17-24. http://dx.doi.org/10.14295/2238-6416.v69i1.302

Silva, L. C. A., Leite, M. O., Vinha, M. B., Brandão, S. C. C., Pinto, C. L. O., \& Carvalho, A. F. (2009). Estudo da qualidade de manteigas por fosfatase alcalina e análises físico-químicas. Revista do Instituto de Latícinios Cândido Tostes, 64(368), $42-47$.

Silveira, M. L. R., \& Bertagnolli, S. M. M. (2014). Avaliação da qualidade do leite cru comercializado informalmente em feiras livres no município de Santa Maria-RS. Revista Visa em Debate, 2(2), 75-80. http://dx.doi.org/10.3395/vd.v2i2.135

Sosnowski, M., Rola, J. G., \& Osek, J. (2016). Alkaline phosphatase activity and microbiological quality of heat-treated goat milk and cheeses. Small Ruminant Research, 136, 132-136. http://dx.doi.org/10.1016/j.smallrumres.2015.12.038 